\title{
Potenziale und Herausforderungen von E-Mental-Health- Interventionen in der Versorgung psychischer Störungen
}

\section{Potentials and Challenges of E-Mental Health Interventions in Mental Health Care}

\author{
Autoren \\ Moritz Köhnen, Jörg Dirmaier, Martin Härter \\ Institute \\ Institut und Poliklinik für Medizinische Psychologie, Zentrum \\ für Psychosoziale Medizin, Universitätsklinikum Hamburg- \\ Eppendorf \\ Schlüsselwörter \\ E-Mental-Health, Internet, online, Versorgung psychischer \\ Gesundheit
}

Key words

e-mental health, internet, online, mental health care

eingereicht 21.12.2018

akzeptiert $\quad 07.02 .2019$

Bibliografie

DOI https://doi.org/10.1055/a-0853-2568

Fortschr Neurol Psychiatr 2019; 87: 160-164

(C) Georg Thieme Verlag KG Stuttgart · New York

ISSN 0720-4299

Korrespondenzadresse

Moritz Köhnen

Universitätsklinikum Hamburg-Eppendorf, Zentrum für

Psychosoziale Medizin - Institut und Poliklinik für

Medizinische Psychologie

Hamburg Martinistr. 52

20246, Hamburg

E-Mail: m.koehnen@uke.de

\section{ZUSAMMENFASSUNG}

Psychische Störungen sind weit verbreitet und führen zu bedeutenden Beeinträchtigungen auf individueller und gesellschaftlicher Ebene. Trotz der starken Krankheitslast und des vergleichsweisen gut ausgebauten Versorgungssystems in Deutschland werden psychische Störungen nur unzureichend versorgt. Auch tragen Zugangsbarrieren wie etwa lange Wartezeiten auf ambulante Behandlungsplätze dazu bei, dass Gesundheitsdienstleistungen nur verzögert oder gar nicht durch Betroffene in Anspruch genommen werden. E-Mental-Health-Interventionen gelten als vielversprechende Optionen, um Zugangsbarrieren abzubauen und
Versorgungslücken, z.B. durch eine Unterversorgung im ländlichen Raum, zu schließen. Sie können zeit- und ortsunabhängig angeboten werden und auch Menschen in Regionen mit geringer Versorgungsdichte oder Menschen mit körperlichen Beeinträchtigungen, die in ihrer Mobilität eingeschränkt sind, erreichen. Trotz des großen Potenzials von E-Mental-Health-Interventionen bestehen zahlreiche Herausforderungen, z. B. in Bezug auf den Datenschutz und die Präzisierung hinsichtlich begrifflicher, konzeptioneller und evaluativer Aspekte des sehr breiten E-(Mental)-HealthSektors. Diese können zum einen hinderlich für die Implementierung in der Routineversorgung sein und zum anderen die Akzeptanz der Interventionen bei Behandlern und Nutzern beeinflussen. Der Überblicksbeitrag beschreibt und diskutiert Potenziale, Herausforderungen und den Präzisierungsbedarf von E-Mental-Health-Interventionen im Kontext der Versorgung psychischer Störungen.

\section{ABSTRACT}

Mental disorders are common and impairing for both affected individuals and society. Despite the great burden of mental disorders and a comparably well-developed mental health care system in Germany, mental disorders are still treated insufficiently. Additionally, access barriers such as long waiting times for outpatient treatment impede utilization of mental health care services. E-mental health interventions are considered as promising options to reduce access barriers and to overcome gaps in the mental health care system. Such interventions can be delivered flexibly (i.e. independent of time and/or space), so that people living in rural and underserved areas, or people with mobility constraints (e.g. because of physical illness or impairment) can be reached adequately. However, the e-(mental) health sector is facing numerous challenges, as there is ambiguity regarding terminological, conceptual and evaluative aspects hindering the implementation process in routine care and affecting acceptance of e-mental health interventions for users and health care providers. Thus, this contribution describes and discusses potentials, challenges and needs for specification (e. g. terminologically) of e-mental health interventions in the context of mental health care. 


\section{Einleitung}

Die Digitalisierung ist nicht nur in unserem Alltag und weiten Teilen des gesellschaftlichen Lebens angekommen - $90 \%$ der deutschen Haushalte besitzen einen Personalcomputer (PC) [1] -, sondern auch zunehmend in der gesundheitlichen Versorgung von Menschen mit psychischen und körperlichen Beschwerden in Deutschland. Auch international betrachtet ist der Trend zu beobachten, dass im Bereich der Gesundheitsversorgung immer häufiger digitale Technologien verwendet werden, um bereits bestehende Versorgungsstrukturen zu unterstützen [2]. Der Einsatz digitaler Technologien in der gesundheitlichen Versorgung hat nicht nur das Potenzial, einzelne Individuen und spezifische Subpopulationen (z. B. Menschen mit körperlichen Beeinträchtigungen) zu erreichen, sondern kann auch den Zugang zur Versorgung in Ländern, welche nur begrenzte Ressourcen besitzen, verbessern [2, 3].

Ganz allgemein beschreibt der Begriff E-Health (Electronic Health) den Einsatz von Informations- und Kommunikationstechnologien (IKT) in gesundheitsrelevanten Feldern. Dabei hat EHealth zum Ziel, die Versorgung zu verbessern und somit die Gesundheit des Individuums und der Gesellschaft zu fördern [4]. Trotz des stetigen Wachstums des E-Health-Sektors (und der zunehmenden Relevanz von E-Health im Forschungskontext) gibt es keine einheitliche Definition des Begriffs E-Health. In der Literatur kursieren daher viele „verwandte“ Begriffe (z. B. „Medicine 2.0“), die stark mit dem Konstrukt E-Health überlappen und z. T. auch austauschbar verwendet werden [5]. Die Weltgesundheitsorganisation (2005) definiert E-Health folgendermaßen: E-Health bezeichnet die kosteneffiziente und sichere Nutzung von IKT zur Förderung von Gesundheit und gesundheitsbezogenen Bereichen; darunter fallen Gesundheitsdienstleitungen, Gesundheitsüberwachung, Gesundheitsliteratur, Gesundheitsbildung sowie Wissen und Forschung (übersetzt nach [6], S. 109).

Der vorliegende Beitrag bezieht sich insbesondere auf E-Health-Anwendungen welche im Kontext der Gesundheitsversorgung psychischer Störungen eingesetzt werden, also dem Bereich E-Mental-Health zuzuordnen sind. Unter E-Mental-Health versteht man den Einsatz von IKT - insbesondere Technologien, die das Internet nutzen, um psychische Gesundheit und psychische Gesundheitsversorgung zu fördern. So werden im Rahmen von E-Mental-Health digitale Technologien eingesetzt, um z. B. frühzeitig Screenings durchzuführen, Gesundheit zu fördern, psychischen Störungen vorzubeugen, sie zu behandeln und eine Rückfallprophylaxe anzubieten. Zusätzlich umfasst der Bereich E-MentalHealth die Verbesserung der Erbringung von gesundheitlichen Dienstleistungen (z. B. über elektronische Patientenakten), die Schulung der Akteure im Gesundheitssystem (E-Learning) sowie die Onlineforschung zur psychischen Gesundheit [7]. Beide Definitionen haben gemeinsam, dass ein sehr breites Feld abgedeckt wird - es reicht von rein administrativen Anwendungen (z. B. elektronische Patientenakte, Krankenhausinformationssysteme) bis zu gesundheitlichen Dienstleistungen (z. B. Fernbehandlungen / -konsultationen, Telemonitoring) und Forschung, die über IKT vermittelt werden. Der Unterschied liegt im Fokus der Definitionen: Erstere bezieht sich auf die Gesundheitsversorgung im Allgemeinen, während sich zweitere insbesondere auf die psychische Gesundheitsversorgung konzentriert.

\section{Charakteristik von internet- und mobilbasierten Interventionen (IMI)}

Internet- und mobilbasierte Interventionen (IMI) sind unterschiedlich ausgestaltet hinsichtlich ihrer technischen Umsetzung, der Theoriebasierung, ihrer Anwendungsbereiche und des Ausmaßes an menschlicher Unterstützung. Sie haben gemeinsam, dass sie an der Veränderung von kognitiven, emotionalen oder behavioralen Prozessen ansetzen und die Anwender unterstützen, erworbene Fähigkeiten mithilfe psychotherapeutischer Techniken in ihrem Alltag zu integrieren [8]. Auch können Interventionen, die auf psychische Beschwerden bei körperlichen Erkrankungen zugeschnitten sind (z. B. Online-Interventionen für Depressionen bei Menschen mit Multiple Sklerose [MS]), über IMI abgebildet werden.

\section{Technische Umsetzung \& Theoriebasierung von IMI}

Der Einsatz von IMI kann über verschiedene technische Medien stattfinden, wie eine Auflistung nach Ebert, Van Daele, Nordgreen und Kollegen [8] zeigt:

1. Darbietung evidenzbasierter Strategien in Form von Selbsthilfe-Sitzungen (z. B. über das Internet).

2. Der Einsatz von virtueller Realität, um beispielsweise eine Konfrontation mit angstbesetzten Situationen im virtuellen Raum durchzuführen.

3. Serious Games, wobei es zum Training von funktionalen psychologischen Techniken kommt.

4. Avatar-gestützte Sitzungen, um z. B. Verhaltensänderungen der Teilnehmer zu fördern.

5. Einsatz von Erinnerungs-, Feedback- und Verstärkungsmechanismen über Apps, E-Mails oder Textnachrichten (SMS), um den Transfer von Interventionsinhalten in den Alltag der Nutzer zu unterstützen.

6. Sensoren in Smartphones und Wearables, oder auch Apps, welche störungsspezifische Symptome, oder gesundheitsbezogene Verhaltensweisen (z. B. Schlafverhalten über Bewegungssensoren, Bewegungsverhalten über Schrittzähler) erfassen und somit unterstützend im Rahmen der Therapie eingesetzt werden können (z. B. zur Motivationsförderung).

Die meisten IMI - speziell wissenschaftlich evaluierte - basieren auf den Grundsätzen der kognitiven Verhaltenstherapie (KVT). IMI können stark strukturiert und standardisiert dargeboten werden (z.B. über automatisierte Online-Programme), weshalb sich manualisierte verhaltenstherapeutische Ansätze besonders gut als theoretische Basis eignen. Darüber hinaus gibt es aber auch andere psychologische bzw. psychotherapeutische Ansätze, wie zum Beispiel achtsamkeitsbasierte (-assozierte) Ansätze, Akzeptanz- und Commitment-Therapie und psychodynamische Ansätze, die als theoretische Grundlage für IMI herangezogen werden können [8].

\section{Anwendungsbereiche}

IMI finden sich in unterschiedlichen Versorgungsbereichen wieder, sie können in der psychischen Gesundheitsförderung und Prävention psychischer Erkrankungen sowie bei der Behandlung psychischer Störungen (z. B. bei unipolaren Depressionen) eingesetzt werden. Zudem gibt es Interventionen, die sich im Bereich der 
Nachsorge ansiedeln lassen und sich zum Beispiel auf die Rückfallprophylaxe psychischer Störungen spezialisiert haben [8].

IMI können im Rahmen einer Stand-Alone-Anwendung (alleinstehende Behandlung), als Teil eines Stepped-Care Ansatzes (stufenbasierte Behandlung) oder in Form von Blended Concepts (z. B. Kombination von Therapie vor Ort und vertiefenden OnlineLektionen) zum Einsatz kommen. Unter Stand-Alone-Anwendung versteht man IMI, die als alleinstehende Intervention ohne zusätzliche Therapiekontakte vor Ort zur Behandlung von psychischen Beschwerden eingesetzt werden. Durch sie kann die Reichweite evidenzbasierter psychologischer Therapieverfahren vergrößert werden, da sie zeit- und/oder ortsunabhängig angeboten werden, zum Beispiel wenn therapeutische Inhalte über das Internet vermittelt werden [8].

Im Rahmen von Blended Concepts werden IMI in Kombination mit klassischer Psychotherapie vor Ort angeboten. IMI können bestimmte Elemente der Therapie vor Ort (z. B. Teile der Psychoedukation) ersetzen, die nicht zwangsläufig über einen Behandler vermittelt werden müssen, folglich bleibt in den Sitzungen vor Ort mehr Zeit, um zum Beispiel Bewältigungsstrategien zu erarbeiten. IMI können aber auch genutzt werden, um die Wirksamkeit der Therapie vor Ort zu erhöhen, indem sie ergänzende Übungsaufgaben (z. B. über eine Onlineplattform) bereitstellen, oder indem sie den Transfer therapeutischer Inhalte in den Alltag der Patienten fördern [8].

Im Rahmen des Stepped-Care-Ansatzes hängt die therapeutische Unterstützung der Patienten von zuvor gemachten Behandlungserfolgen / -misserfolgen ab. IMI können zum Beispiel bei der Behandlung von Depressionen in Form von (un)angeleiteter Selbsthilfe - vermittelt über das Internet - eingesetzt werden, sie dienen dabei als erster Schritt in der Behandlungskette von Menschen mit einer depressiven Störung. Wenn die Patienten nicht adäquat (z. B. Symptomreduktion) auf die Behandlung reagieren, wird die Behandlung im Stepped-Care-Modell angepasst (sog. „Step-Up”) und intensivere Interventionen wie engmaschige, ambulante Psychotherapie oder Medikamente kommen zum Einsatz. Gleiches ist auch in die andere Richtung möglich, zum Beispiel können Patienten nach einer erfolgreichen Akutbehandlung heruntergestuft werden (sog. "Step-Down"), sodass anschließend IMI zur Sicherung der Rückfallprophylaxe zum Einsatz kommen $[8,9]$.

\section{Ausmaß menschlicher Unterstützung}

Das Ausmaß menschlicher Unterstützung kann je nach Intervention sehr unterschiedlich aussehen. Aktuell ist die Methode der angeleiteten Selbsthilfe am weitesten verbreitet, hierbei werden evidenzbasierte Inhalte, die weitestgehend selbständig bearbeitet werden können, dargeboten. Zusätzlich wird regelmäßiges, meist wöchentliches Feedback oder Beratung angeboten. Dabei liegt der Schwerpunkt der menschlichen Unterstützung - insbesondere bei StandAlone-Anwendungen - oftmals in der Förderung der Adhärenz an die Intervention, z.B. durch die Motivation der Nutzer per Mail oder Chat, und seltener in der Vermittlung psychotherapeutischer Inhalte. Menschliche Unterstützung kann entweder über synchrone oder asynchrone (d. h. in Echtzeit, z. B. über das Telefon) Kommunikationswege (d. h. zeitlich verzögert, z. B. über E-Mail) stattfinden. Dabei ist der Einsatz asynchroner Kommunikation weitaus häufiger vertreten, je nach Intervention kann es zudem sehr unterschiedlich sein, wie viel Zeit beispielsweise ein begleitender Therapeut zur Unterstützung der Nutzer aufwendet [8].

\section{Chancen und Herausforderungen in der Versorgung von psychischen Störungen}

E-Mental-Health-Interventionen im Allgemeinen und IMI im Speziellen haben das Potenzial, die Versorgung von Menschen mit psychischen Beschwerden zu verbessern, da sie weitestgehend zeit- und/oder ortsunabhängig angeboten werden. So können Menschen in versorgungschwachen Regionen (z.B. Iändlicher Raum) oder auch Menschen mit körperlichen Einschränkungen besser erreicht werden [10]. Auch können Menschen, die traditionelle Behandlungsangebote ablehnen und es vorziehen, anonym zu bleiben, z. B. aus Furcht vor Stigmatisierung, von derartigen Angeboten profitieren [11]. Zusätzlich ist die Wirksamkeit von IMI für viele psychische Störungen (z. B. Depressionen, Angststörungen) über methodisch hochwertige Studien nachgewiesen [12, 13]. Angesichts langer Wartezeiten in der ambulanten psychotherapeutischen Versorgung [14] werden IMI häufig als aussichtsreiche Option diskutiert, um Wartezeiten zu überbrücken. Die Überbrückung von Wartezeiten kann das Risiko einer Chronifizierung psychischer Störungen reduzieren - bspw. durch frühzeitige ... Symptomreduktion. Zudem ist es möglich, im Rahmen einer vorgeschalteten niederschwelligen Intervention (z. B. über das Internet) auf die anschließende psychotherapeutische Behandlung vorzubereiten. Allerdings gibt es bisher nur wenige Studien, die derartige Interventionen zur Überbrückung von Wartezeiten untersucht haben [z. B. 15, 16]. Daher ist es unklar, ob und vor allem wie IMI diesen Versorgungsbereich sinnvoll ergänzen können. IMI ohne menschliche Begleitung scheinen aufgrund ihrer geringen Adhärenz wenig geeignet, um Wartezeiten in der ambulanten psychotherapeutischen Versorgung zu überbrücken [15]. Eine Herausforderung besteht darin, IMI für diesen Versorgungskontext so zu gestalten, dass sie in angemessenem Maße genutzt werden und ihre Wirksamkeit entfalten können. Insgesamt besitzen IMI also das Potenzial, die Behandlung von psychischen Beschwerden flexibler zu gestalten (z. B. ortsunabhängige Behandlung), Versorgungslücken zu schließen und Stigmatisierungsbefürchtungen abzubauen.

Trotz des großen Potenzials gibt es auch Hürden, die es zu überwinden gilt, um IMI in der Versorgung zu implementieren. Zum einen sind datenschutzrechtliche Fragen zu klären (z. B. Verarbeitung von Nutzerdaten, gesicherte Verbindung zwischen Behandler und Patient) [8, 17]. Dazu kommt, dass es bisher keine einheitlich geltenden Qualitätsstandards für IMI gibt, an denen sich Nutzer oder andere Akteure des Gesundheitssystems orientieren können [18]. Bestehende Forschungslücken stellen auch eine mögliche Hürde für die Umsetzung dar. Obwohl für bestimmte psychische Störungen (z. B. Depressionen oder Angststörungen) vielfach Wirksamkeitsnachweise zu IMI vorliegen, gibt es nur wenige Studien, die potenzielle Risiken und Nebenwirkungen, wie Behandlungsabbrüche und Symptomverschlechterungen von IMI untersuchten [19].

Zusammenfassend zeigt sich, dass IMI vielfältige Optionen bieten, um die Versorgung psychischer Störungen sinnvoll zu 
ergänzen. Dennoch müssen Barrieren der Umsetzung überwunden werden, sodass IMI ihr volles Potenzial entfalten können. Auch wird deutlich, dass die Implementierung eher eine gesamtgesellschaftliche Aufgabe darstellt, bei der unterschiedliche Akteure (z. B. Forschung, Politik, Wirtschaft, Behandler, Nutzer) beteiligt sind, welche Implementierungsbestrebungen entweder erleichtern oder erschweren können.

\section{IMI bei chronischen somatischen Erkrankungen}

IMI kommen nicht nur bei psychischen Störungen zum Einsatz, sondern auch wenn im Kontext somatischer Erkrankungen psychische Belastungen oder Störungen auftreten. Beispielsweise gibt es Studien, die die Wirksamkeit von IMI bei Menschen mit Psoriasis [20], Diabetes [21], chronisch obstruktiver Lungenerkrankung [22], Migräne [23], Herzkreislauferkrankungen [24] und onkologischen Erkrankungen untersuchten [25]. Auf Basis systematischer Übersichtarbeiten (d.h. unter Einsatz von Verfahren zur Evidenzsynthese) sind IMI zum Beispiel für chronische somatische Erkrankungen im Allgemeinen [26, 27], MS [28], Krebsüberlebende [29-31] und Brustkrebspatientinnen [32, 33] untersucht worden. Diese Arbeiten zeigen, dass IMI psychische Belastungen, wie (Krebs-bezogene) Fatigue, und andere gesundheitsrelevante Endpunkte (z. B. Depressionen, gesundheitsbezogene Lebensqualität) positiv beeinflussen [29-31]. Zudem zeigte sich, dass therapeutisch unterstützte E-Mental-HealthInterventionen wirksamer in der Reduktion von Fatigue sind als unbegleitete Interventionen [31]. Im Allgemeinen können chronische somatische Erkrankungen erfolgreich mit IMI - insbesondere mit internetbasierter kognitiver Verhaltenstherapie behandelt werden. IMI können sowohl psychologische (depressive Symptome, Ängste, Stress) als auch störungsspezifische Belastungen (z. B. Kopfschmerz, störungsspezifischer Stress) bei chronischen somatischen Erkrankungen reduzieren [26].

Trotz des Vorliegens von systematischen Übersichtarbeiten ist die Wirksamkeit, Akzeptanz und Sicherheit von E-MentalHealth-Interventionen bei vielen somatischen Erkrankungen und damit einhergehenden psychischen Belastungen noch nicht ausreichend geklärt. Dies liegt zum einen an der geringen methodischen Qualität einzelner Studien und zum anderen daran, dass bestimmte Erkrankungen nur unzureichend untersucht sind [27].

\section{FAZIT}

Laut Weltgesundheitsorganisation ist E-Health kein „Add-on“, sondern eine zentrale und essentielle Maßnahme, die zur Verbesserung der gesundheitlichen Versorgung im 21sten Jahrhundert beitragen kann [2]. Trotz des großen Potenzials von E-Health (und E-Mental-Health) gibt es noch zahlreiche Herausforderungen und ungeklärte Fragen, die eine Umsetzung in der Versorgung einschränken:

- E-Mental-Health-Interventionen bieten Patienten den Vorteil, dass sie psychische Versorgungsleistungen weitestgehend unabhängig von Ort und Zeit in Anspruch nehmen können, sodass auch Menschen mit körperlichen Beeinträchtigungen oder Menschen, die im ländlichen Raum wohnen, erreicht werden. Auch Menschen in besonderen Lebenslagen (beruflich oder privat) können von der Flexibilität derartiger Interventionen profitieren.

- E-Mental-Health-Interventionen besitzen ein großes Potenzial für Patienten, die neben einer somatischen Erkrankung auch psychische Beschwerden erleben und sind deshalb medizinpsychologisch besonders interessant und relevant. Sie können sowohl psychische (z. B. Stress, Depression) als auch erkrankungsbezogene Belastungen (z. B. Kopfschmerzfrequenz) reduzieren und die Lebensqualität der Betroffenen bedeutend verbessern.

- Aus ethischer Perspektive wäre es bedenklich, wenn erwiesenermaßen wirksame Interventionen nicht den Weg in die Versorgung Betroffener finden würden. Andererseits besteht die Gefahr, dass E-Mental-Health-Interventionen nicht eingesetzt werden, weil sie wirksam sind, sondern lediglich um bestehende Lücken in der Versorgung (z. B. im ländlichen Raum) zu schließen. E-MentalHealth-Interventionen können eine erste Lösung darstellen, um Versorgungsengpässen zu begegnen. Allerdings bedarf es auch Personal vor Ort, was einen multimodalen Lösungsansatz unterstreicht.

Interessenkonflikt

Die Autoren geben an, dass kein Interessenkonflikt besteht.

\section{Literatur}

[1] Statistisches Bundesamt. Ausstattung mit Gebrauchsgütern. Im Internet: https://www.destatis.de/DE/ZahlenFakten/GesellschaftStaat/Ein kommenKonsumLebensbedingungen/AusstattungGebrauchsguetern/ Tabellen / A_Infotechnik_D_LWR.html; Stand: 2018 Oct 05

[2] World Health Organization. Global diffusion of eHealth: making universal health coverage achievable: report of the third global survey on eHealth. Geneva: World Health Organization; 2016

[3] Marzano L, Hollis C, Cipriani A et al. Digital technology: coming of age? Evid Based Ment Health 2017; 20: 97. doi:10.1136/eb-2017-102821

[4] Fischer F, Aust V, Krämer A. eHealth: Hintergrund und Begriffsbestimmung. In: Fischer F, Krämer A, Hrsg. eHealth in Deutschland: Anforderungen und Potenziale innovativer Versorgungsstrukturen. Berlin, Heidelberg: Springer-Verlag; 2016: 3-23

[5] Boogerd EA, Arts T, Engelen L] et al. "What Is eHealth": time for an update? JMIR Res Protoc 2015; 4: e29. doi:10.2196/resprot.4065

[6] World Health Organization. Resolution WHA58.28. eHealth. In: Fiftyeighth World Health Assembly, Geneva, 16-25 May 2005. Resolutions and decisions annex. Geneva: World Health Organization; 2005

[7] Riper H, Andersson G, Christensen $\mathrm{H}$ et al. Theme issue on e-mental health: a growing field in internet research. J Med Internet Res 2010; 12: e74. doi:10.2196/jmir.1713

[8] Ebert DD, Van Daele T, Nordgreen T et al. Internet-and Mobile-Based Psychological Interventions: Applications, Efficacy, and Potential for 
Improving Mental Health. Eur Psychol 2018; 23: 167-187. doi:10.1027| 1016-9040/a000318

[9] Härter M, Watzke B, Daubmann A et al. Guideline-based stepped and collaborative care for patients with depression in a cluster-randomised trial. Sci Rep 2018; 8: 9389. doi:10.1038/s41598-018-27470-6

[10] Benavides-Vaello S, Strode A, Sheeran BC. Using technology in the delivery of mental health and substance abuse treatment in rural communities: a review. J Behav Health Serv Res 2013; 40: 111-120. doi:10.1007| s11414-012-9299-6

[11] Townsend L, Gearing RE, Polyanskaya O. Influence of health beliefs and stigma on choosing internet support groups over formal mental health services. Psychiatric Services 2012; 63: 370-376. doi:10.1176/appi. ps.201100196

[12] Kampmann IL, Emmelkamp PMG, Morina N. Meta-analysis of technology-assisted interventions for social anxiety disorder. J Anxiety Disord 2016; 42: 71-84. doi:10.1016/j.janxdis.2016.06.007

[13] Königbauer J, Letsch J, Doebler $P$ et al. Internet- and mobile-based depression interventions for people with diagnosed depression: A systematic review and meta-analysis. J Affect Disord 2017; 223: 28-40. doi:10.1016/j.jad.2017.07.021

[14] Bundespsychotherapeutenkammer. Ein Jahr nach der Reform der Psychotherapie-Richtlinie - Wartezeiten 2018. Im Internet: https: / /www. bptk.de/uploads/media/20180411_BPtK-Studie_Wartezeiten_2018.pdf; Stand: 2018 Nov 26

[15] Fuhr K, Fahse B, Hautzinger $M$ et al. Erste Erfahrungen zur Implementierbarkeit einer internet-basierten Selbsthilfe zur Überbrückung der Wartezeit auf eine ambulante Psychotherapie. Psychother Psychosom Med Psychol 2018; 68: 234-241. doi:10.1055/s-0043-122241

[16] Kenter RM, Cuijpers P, Beekman A et al. Effectiveness of a Web-Based Guided Self-help Intervention for Outpatients With a Depressive Disorder: Short-term Results From a Randomized Controlled Trial. J Med Internet Res 2018; 18: e80. doi:10.2196/jmir.4861

[17] Torous J, Nicholas J, Larsen ME et al. Clinical review of user engagement with mental health smartphone apps: evidence, theory and improvements. Evid Based Ment Health 2018; 21: 116-119. doi:10.1136/eb2018-102891

[18] Klein J, Gerlinger G, Knaevelsrud C et al. Internet-based interventions in the treatment of mental disorders: Overview, quality criteria, perspectives. Nervenarzt 2016; 87: 1185-1193. doi:10.1007/s00115-016-0217-7

[19] Ebert DD, Baumeister $\mathrm{H}$. Internet-und mobilbasierte Interventionen in der Psychotherapie: ein Überblick. Psychotherapeutenjournal 2016; 1: 22-31

[20] Bundy C, Pinder B, Bucci S et al. A novel, web-based, psychological intervention for people with psoriasis: the electronic Targeted Intervention for Psoriasis (eTIPs) study. Br J Dermatol 2013; 169: 329-336. doi: doi:10.1111/bjd.12350

[21] Nobis S, Lehr D, Ebert DD et al. Efficacy of a Web-Based Intervention With Mobile Phone Support in Treating Depressive Symptoms in Adults
With Type 1 and Type 2 Diabetes: A Randomized Controlled Trial. Diabetes Care 2015; 38: 776-783. doi:10.2337/dc14-1728

[22] Walters ], Cameron-Tucker $\mathrm{H}$, Wills $\mathrm{K}$ et al. Effects of telephone health mentoring in community-recruited chronic obstructive pulmonary disease on self-management capacity, quality of life and psychological morbidity: a randomised controlled trial. BMJ Open 2013; 3: e003097. doi:10.1136/bmjopen-2013-003097

[23] Hedborg K, Muhr C. Multimodal behavioral treatment of migraine: An Internet-administered, randomized, controlled trial. Ups J Med Sci 2011; 116: 169-186. doi:10.3109/03009734.2011.575963

[24] Glozier N, Christensen H, Naismith S et al. Internet-Delivered Cognitive Behavioural Therapy for Adults with Mild to Moderate Depression and High Cardiovascular Disease Risks: A Randomised Attention-Controlled Trial. PLoS One 2013; 8: e59139. doi:10.1371/ journal.pone.0059139

[25] Willems RA, Bolman CAW, Mesters I et al. Short-term effectiveness of a web-based tailored intervention for cancer survivors on quality of life, anxiety, depression, and fatigue: randomized controlled trial. Psychooncology 2017; 26: 222-230. doi:doi:10.1002/pon.4113

[26] van Beugen S, Ferwerda M, Hoeve D et al. Internet-based cognitive behavioral therapy for patients with chronic somatic conditions: a meta-analytic review. J Med Internet Res 2014; 16: e88. doi:10.2196/ jmir.2777

[27] Sharp J, Holly D, Broomfield N. Computerized cognitive behaviour therapy for depression in people with a chronic physical illness. $\mathrm{Br}$ ] Health Psychol 2013; 18: 729-744. doi:10.1111/bjhp.12014

[28] Proctor B], Moghaddam N, Vogt W et al. Telephone psychotherapy in multiple sclerosis: A systematic review and meta-analysis. Rehabil Psychol 2018; 63: 16. doi:10.1037/rep0000182

[29] Post KE, Flanagan J. Web based survivorship interventions for women with breast cancer: An integrative review. Eur J Oncol Nurs 2016; 25 : 90-99. doi:10.1016/j.ejon.2016.10.004

[30] Kim AR, Park H-A. Web-based Self-management Support Interventions for Cancer Survivors: A Systematic Review and Meta-analyses. Medinfo 2015; 216: 142-147. doi:10.3233/978-1-61499-564-7-142

[31] Seiler A, Klaas V, Tröster G et al. eHealth and mHealth interventions in the treatment of fatigued cancer survivors: A systematic review and meta-analysis. Psychooncology 2017; 26: 1239-1253. doi:10.1002/ pon. 4489

[32] Zhang Q, Zhang L, Yin R et al. Effectiveness of telephone-based interventions on health-related quality of life and prognostic outcomes in breast cancer patients and survivors - A meta-analysis. Eur J Cancer Care 2018; 27: e12632

[33] Chen Y-Y, Guan B-S, Li Z-K et al. Effect of telehealth intervention on breast cancer patients' quality of life and psychological outcomes: a meta-analysis. J Telemed Telecare 2018; 24: 157-167. doi:10.1177| $1357633 \times 16686777$ 